\title{
BMJ Assessment of statin-associated muscle open toxicity in Japan: a cohort study conducted using claims database and laboratory information
}

\author{
Chia-Hsien Chang, ${ }^{1,2}$ Makiko Kusama, ${ }^{2}$ Shunsuke Ono, ${ }^{2}$ Yuichi Sugiyama, ${ }^{2,3}$ \\ Takao Orii, ${ }^{4}$ Manabu Akazawa ${ }^{5}$
}

To cite: Chang $\mathrm{C}-\mathrm{H}$, Kusama M, Ono S, et al. Assessment of statinassociated muscle toxicity in Japan: a cohort study conducted using claims database and laboratory information. BMJ Open 2013;3:e002040.

doi:10.1136/bmjopen-2012002040

- Prepublication history for this paper are available online. To view these files please visit the journal online (http://dx.doi.org/10.1136/ bmjopen-2012-002040).

Received 31 August 2012 Revised 28 February 2013 Accepted 18 March 2013

This final article is available for use under the terms of the Creative Commons Attribution Non-Commercial 2.0 Licence; see http://bmjopen.bmj.com

For numbered affiliations see end of article.

\section{Correspondence to} Professor Manabu Akazawa; makazawa@my-pharm.ac.jp

\begin{abstract}
Objective: To estimate the incidence of muscle toxicity in patients receiving statin therapy by examining study populations, drug exposure status and outcome definitions.

Design: A retrospective cohort study.

Setting: 16 medical facilities in Japan providing information on laboratory tests performed in and claims received by their facilities between 1 April 2004 and 31 December 2010.
\end{abstract}

Participants: A database representing a cohort of 35903 adult statin (atorvastatin, fluvastatin, pitavastatin, pravastatin, rosuvastatin and simvastatin) users was studied. Use of interacting drugs (fibrates, triazoles, macrolides, amiodarone and ciclosporin) by these patients was determined.

Main outcome measure: Statin-associated muscle toxicity (the 'event') was identified based on a diagnosis of muscle-related disorders (myopathy or rhabdomyolysis) and/or abnormal elevation of creatine kinase (CK) concentrations. Events were excluded if the patients had CK elevation-related conditions other than muscle toxicity. Incidence rates for muscle toxicity were determined per 1000 person-years, with $95 \% \mathrm{Cl}$ determined by Poisson regression.

Results: A total of 18036 patients accounted for 42193 person-years of statin therapy, and 43 events were identified. The incidence of muscle toxicity in the patients treated with statins was $1.02(95 \% \mathrm{Cl} 0.76$ to $1.37) / 1000$ person-years. The estimates varied when outcome definitions were modified from $0.09 / 1000$ person-years, which met both diagnosis and CK 10x greater than the upper limit of normal range (ULN) criteria, to 2.06/1000 person-years, which met diagnosis or CK $5 \times$ ULN criterion. The incidence of muscle toxicity was also influenced by the statin therapies selected, but no significant differences were observed. Among 2430 patients (13.5\%) received interacting drugs with statins, only three muscle toxicity cases were observed (incidence: 1.69/1000 person-years).

Conclusions: This database study suggested that statin use is generally well tolerated and safe; however, the risk of muscle toxicity related to the use of interacting drugs requires further exploration.

\section{ARTICLE SUMMARY}

Article focus

- Asian populations are more sensitive to statins' clinical response than Western populations.

- Our objective was to evaluate the risk of muscle toxicity associated with statin and/or interacting drug use, by using claims database and laboratory information in Japan.

Key messages

- Patients receiving prescription statins were most often prescribed the lower limits of approved dosages, with rare concomitant use of interacting drugs that could increase steady-state statin concentrations.

- The number of adverse events was limited; the incidence of muscle toxicity was statistically indistinguishable among statins.

- This study has major implications for risk evaluation, given the information on infrastructure available to validate claims-based estimations.

Strengths and limitations of this study

- This is the first study to evaluate drug-associated risk by using claims database and laboratory information in Japan, indicating the potential applicability of electronic health information as a resource for applied analyses.

- Interacting drug use associated with increased risk of muscle toxicity requires further exploration because a low number of person-years of observations currently exists in the literature. Diagnostic information is slightly less complete than is prescription and laboratory test information; researchers should use caution when interpreting related information in database studies.

\section{INTRODUCTION}

Statins (3-hydroxy-3-methyl-glutaryl coenzyme A reductase inhibitor or HMG-coenzyme A reductase inhibitor) are widely used for adults presenting with cardiovascular disease (CVD), and those with a $20 \%$ or greater 10 -year risk of developing $\mathrm{CVD},{ }^{1}$ to reduce 
the incidence of cardiovascular comorbidity and mortality. Although statins are well tolerated by a vast majority of patients, their use can lead to infrequent adverse muscle, renal and hepatic events. ${ }^{2}$ Severe adverse events could lead to additional drug costs, increasing the burden of healthcare expenditures. ${ }^{4}$ In addition, the concomitant use of interacting drugs could increase the risk of muscle toxicity. ${ }^{5}$ Moreover, the Asian population is more sensitive in its clinical response to statins than is the Western population, and approved statin doses in Japan are relatively low compared with those approved for use in the USA. ${ }^{6-9}$

Clinical trials are restricted in the number and diversity of participants enrolled, such that the chances of detecting rare adverse treatment effects are low. Regulatory bodies, including those in Japan, have long relied primarily on the voluntary reporting system to monitor postmarketing safety. In addition to voluntary reporting, which does not accurately reflect risk due to under-reporting, a major challenge for assessing the safety of statin use is a lack of comparative data. Given concerns about the limitations of existing monitoring systems, the use of automatic databases such as claims or electronic healthcare records for postmarketing safety assessment has been well structured and widely applied in the USA and European Union. ${ }^{10}{ }^{11}$ Recently, the Sentinel System was launched by the US Food and Drug Administration (FDA) to develop active surveillance capabilities for evaluating postmarket safety issues in regulated medical products. ${ }^{12}$ In Japan, in order to complement this strategy for safety assessment, the Medical Information of Risk Assessment Initiative (MIHARI project) was launched by the Pharmaceutical and Medical Devices Agency in 2009. ${ }^{13}{ }^{14}$ In retrospective studies, a health outcome was defined by criteria that are restricted by the structure of a given database. Different definitions for explaining a certain adverse event could result in different conclusions. Before researchers and regulators begin working with large automated databases (including the MIHARI database) for drug safety monitoring, pilot studies are needed to evaluate the pros and cons of database studies under the Japanese healthcare system.

In early 2012, the FDA issued labelling changes for statin drugs based on updated safety information. ${ }^{15}$ Administrative databases may be useful for identifying potential problems, such as assessment of risk factors and incidence of adverse treatment effects. Our study evaluated the rare adverse event of statin-associated muscle toxicity (eg, myopathy or rhabdomyolysis), defined by two criteria, by using the claims database with laboratory information.

\section{METHODS AND ANALYSIS}

\section{Data resource}

This retrospective cohort study analysed data from Medical Data Vision Co Ltd (MDV) in Tokyo, Japan. This commercial, electronic, record-based healthcare database provides information on ambulatory service, hospitalisation, medication use and laboratory tests for patients from 1 January 2004 through 31 December 2010. It contains the patients' demographic characteristics (eg, age and sex), diagnoses (International Statistical Classification of Disease and Related Health Problems (ICD-10 codes)), prescription information (dose, quantity and number of days of supply) and the results of laboratory tests for approximately 410000 patients at 16 medical facilities across Japan. ${ }^{16}$ Although the source of information was limited to 16 facilities, the age and gender distribution of patients in the database was similar to that of the national demographics. Furthermore, the database had been used for various epidemiological studies including an observational study examining a national estimate of acute pancreatitis risk among diabetes patients in Japan. ${ }^{17}$ Patients' identities have been encrypted for protection of privacy, but the data sets could be linked using unique, anonymous identifiers created by the data providers for research purposes. This study was approved by the Institutional Review Board at Meiji Pharmaceutical University and was conducted in compliance with the Japanese Ethical Guidelines for Epidemiological Research, updated in December 2008. ${ }^{18}$

\section{Study cohort}

Patients aged 18 or above who received initiated statin therapy between 1 July 2004 and 30 June 2010 were included in this study. As the median duration of statin prescription in the database was 28 days, a patient would be enrolled if there was no existing claim of a statin prescription within 3 months after the first date of any claim. To avoid enrolling prevalent cases with muscle toxicity, patients who exhibited the following conditions during the 3-month period prior to statin initiation would be excluded: a diagnosis of rhabdomyolysis/myositis; or the possibility of muscle-related CK elevation (ie, patients whose CK elevation did not present with myocardial infarction, myocarditis, trauma or hypothyroidism, and who had no claims of obtaining nitrate or levothyroxine prescriptions within 3 days after concurrent elevation of $\mathrm{CK})$. The eligible population was required to have undergone at least one blood test during statin therapy, to construct a cohort in which ascertainment of outcome was potentially equivalent.

\section{Exposure of interest}

The information on statin therapy was extracted from the claims database, and exposure time was estimated for each patient based on the amount of statin continuously received by the patient, reported as person-years. We assumed that the patients received consecutive treatment from the initiation of the statin therapy until the end of the last prescription, because the patients were monitored regularly. ${ }^{19} 20$ The statins commercially available in Japan during the investigation period were atorvastatin, fluvastatin, pitavastatin, pravastatin, rosuvastatin and 
simvastatin. The prescribing dosage was defined as the sum total of the doses prescribed during the follow-up period, divided by exposure time. As medications might be changed or added for treatment purposes, patients were allowed to contribute to multiple cohorts.

We also defined drugs that have some pharmacokinetic interactions with statins and that may increase the risk of muscle toxicity. Lists of potentially interacting drugs were compiled from package inserts for statin medications, excluding topical and ophthalmic preparations. ${ }^{21}$ We determined whether patients had used any of the following interacting drugs while undergoing the statin therapy: fibrate (benzafibrate, fenofibrate and clinofibrate), macrolide antibiotics (clarithromycin, erythromycin and telithromycin), triazole antimycotics (fluconazole, itraconazole, fosfluconazole and voriconazole), immunosuppressant (cyclosporine), antiarrhythmic drug (amiodarone) and HIV/AIDS drugs (saquinavir/ritonavir, atazanavir, etravirine and efavirenz). Statin therapy administrated concurrently with an interacting agent was treated as a time-varying covariate. Subjects could contribute persontime to statin both with and without a concomitant interacting drug. As the package inserts list the aforementioned interacting drugs as contraindicated or to be used with caution, concomitant use of these drugs with statins is rare. Therefore, we pooled all interacting drugs together rather than assessing specific drug interactions.

\section{Case identification}

Previous studies generally defined statin-associated muscle toxicity by diagnosis and/or by elevated creatine kinase (CK) concentration. ${ }^{22}{ }^{23}$ Since databases based on insurance claims do not contain laboratory results, differences in risk estimation would occur with the varying composition of available data. Therefore, we identified muscle toxicity by using the following two criteria to explore the deviation between differing definitions. Criterion A was based on diagnosis of muscle-related disorders. As there is no specific ICD-10 code to indicate muscle toxicity, the diagnosis contained the words 'myositis' or 'rhabdomyolysis', which were originally written in the Japanese language and were identified using a computer-assisted text searching method (FINDW function in SAS). Criterion $B$ was based on laboratory results. A patient whose CK concentration was greater than 10 times the upper limit of the normal range under statin therapy would be identified as a case. The normal range was given according to the sensitivity of the reference agents used in laboratory tests at each medical facility. The case was recognised as an event if no disease-related condition accompanied by CK elevation was confirmed. The disease-related conditions for CK elevation were as follows: any presence of diagnosis for myocardial infarction, myocarditis, trauma or hypothyroidism, and any claim of nitrate and levothyroxine prescriptions being obtained within 3 days after the muscle toxicity event. ${ }^{24}$ Statin therapy might be discontinued upon development of intolerable muscle symptoms, with or without CK elevation, in patients for whom other aetiologies were ruled out. ${ }^{24}$ Physicians' management decisions about continuation or discontinuation of treatment were determined by compiling claims from patients' health records. Discontinuation and switching were defined as no use of statin therapy or initiation of another statin therapy in the 6 months following occurrence of muscle toxicity.

\section{Statistical analyses}

The incidence rate for muscle toxicity was presented per 1000 person-years with 95\% CI estimated by Poisson regression. The baseline period was defined as 180 days prior to statin initiation. Demographic (age, gender) and coadministration data were extracted from medical claims from the baseline period within the statin inception cohort. Patients were observed until occurrence of the first muscle toxicity event, and were censored when statin therapy was discontinued or when the end of the observational period was reached (31 December 2010). In addition to claims data, we identified the presence of comorbidities from laboratory information for the baseline period. Renal impairment was defined by serum creatine concentrations above the upper limit of the normal range; hepatic impairment was indicated where laboratory values for alanine transaminase and aspartate transaminase increased by more than three times the upper limit of the normal during the baseline period. The cut-off of glycated haemoglobin (HbAlc) level for diabetes mellitus was set at greater than $6.1 \%$, according to diagnostic criteria adopted in Japan. ${ }^{25}$ To compare the characteristics of demographic and clinical variables between criteria, Kolmogorov-Smirnov tests were performed for continuous variables, and $\chi^{2}$ tests were used for dichotomous variables. Comorbidity and coadministration of drugs were defined according to whether claims of a prescription were made prior to 180 days before occurrence of an event. In addition, to determine the muscle toxicity risk associated with drug-drug interactions, the incidence of muscle toxicity among patients who had concomitantly used interacting drugs was compared with that in patients who did not. Various sensitivity analyses were performed by modifying baseline periods (6 and 12 months), statin exposure status (number of days on which statins were received as a denominator) and outcome definitions (abnormal range of CK values and switching, renal dysfunction or hospitalisation, with muscle toxicity). All statistical analyses were carried out using SAS software V.9.3 (SAS Institute Inc, 2012, Cary, North Carolina, USA).

\section{RESULTS}

A total of 18036 patients met all the criteria to be defined as new statin users (figure 1). Of these patients, 11468 $(64 \%)$ were diagnosed as having dyslipidaemia, 14355 $(80 \%)$ had higher than normal levels of cholesterol, triglyceride or low-density lipoprotein and 9382 (52\%) were 
Figure 1 Flow chart of the study cohort.

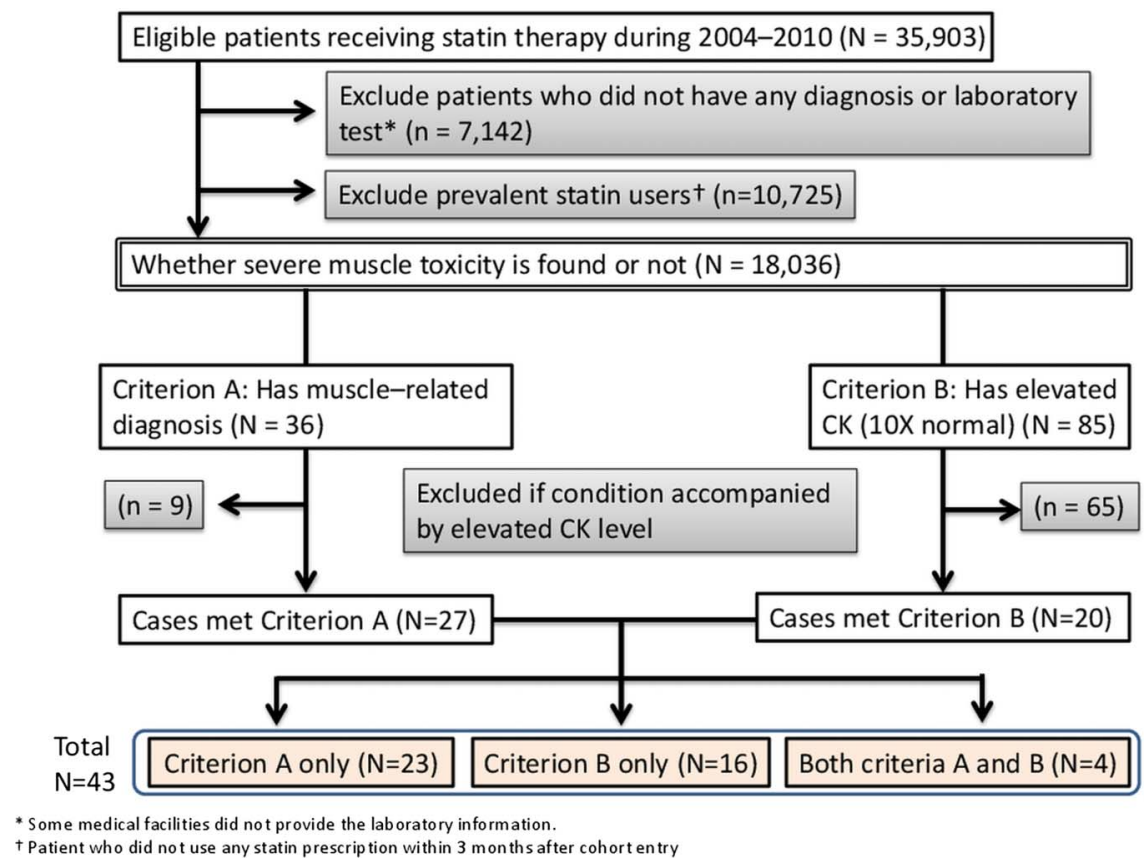

diagnosed as having dyslipidaemia accompanied by higher than normal lipid levels. The mean (SD) age and follow-up month of patients in whom statin use was initiated were 66 (12) years and 29 (22) months, respectively, and $45 \%$ of patients were males. Atorvastatin was the most prevalent HMG-coenzyme A reductase inhibitor and contributed to $37 \%$ of the total person-years in the inception cohort. The prescribed dosage was generally around the lower limit of the approved dose, with limited variation, regardless of the statin (table 1 ).

Among the new statin users, 43 (0.24\%) who met either criterion A or B were identified as base cases, and incidence (with 95\% CI) of muscle toxicity was estimated as $1.02(0.76$ to 1.37$) / 1000$ person-years (table 2). According to various outcome definitions, the estimated incidences ranged from 0.09 (met both criteria A and B) to 2.06 (either met criterion A or demonstrated CK values $>5 \times$ ULN) $/ 1000$ person-years. When the strictest definition was selected (ie, cases met both criteria A and B), four cases were identified, and most of these showed discontinuation, switching, renal dysfunction or hospitalisation after the occurrence of the adverse event (3, 1, 3 and 4 cases, respectively). No significant changes were observed when baseline periods or statin exposure status were modified. Incidence of muscle toxicity in the patients treated with statin monotherapy ranged from 0.45 with pravastatin to 1.73 with rosuvastatin per 1000 person-years (table 3). Using atorvastatin, the most widely prescribed statin in Japan, as the reference, incidences of muscle toxicity were statistically indistinguishable among the statins, regardless of the criterion used. Similarly, demographic and other characteristics of the cases did not differ for the two criteria (table 4).

Of new statin users, 2430 (13.5\%) received interacting drugs during the follow-up period. Regarding the incidence rate of muscle toxicity with respect to interacting drug use, a low number of person-years of observation were contributed, representing limited use of interacting drugs, even when all statins and interacting drugs were aggregated. Owing to the low number of events recorded, we found the wide $95 \%$ CI included the estimated incidence rate (table 5).

\section{DISCUSSION}

This study extends previous work in measuring statin-associated muscle toxicity by using the claims database with laboratory information for actual patient records in Japan. Since the package insert suggests periodical laboratory tests during statin therapy, it is possible to assess the change of medical condition. With an estimated incidence of approximately 1/1000 person-years of statin use, a low occurrence of muscle toxicity was found in the present study. Moreover, less than about one in five patients was found to show concomitant use of interacting drugs, implying that the use of statins is generally well tolerated and safe in Japan. The characteristics and incidences of muscle toxicity between statins were not significantly different. In addition, clinical characteristics were similar among cases defined by diagnosis and cases defined by laboratory results. However, the number of cases in which dyslipidaemia was diagnosed was slightly lower than the number of cases with concurrent elevated lipid levels, indicating an inconsistency between diagnosis and laboratory data.

Information on the frequency ( $\%$ of users) of muscle toxicity is not provided in Japanese statin medication package inserts, with the exceptions of those of rosuvastatin $(0.1 \%)$ and simvastatin $(0.01 \%)$. The lower limit of the $95 \%$ CI showed a value consistent with available package inserts; however, it is possible that adverse events would occur more frequently with widespread use 
Table 1 Characteristics of new statin users $(\mathrm{N}=18036)$ for six drugs represented in this study

\begin{tabular}{|c|c|c|c|c|c|c|}
\hline & $\begin{array}{l}\text { Atorvastatin } \\
(\mathrm{N}=7052)\end{array}$ & $\begin{array}{l}\text { Rosuvastatin } \\
(\mathrm{N}=5921)\end{array}$ & $\begin{array}{l}\text { Pravastatin } \\
(\mathrm{N}=5110)\end{array}$ & $\begin{array}{l}\text { Pitavastatin } \\
(\mathrm{N}=1774)\end{array}$ & $\begin{array}{l}\text { Simvastatin } \\
(\mathrm{N}=976)\end{array}$ & $\begin{array}{l}\text { Fluvastatin } \\
(\mathrm{N}=871)\end{array}$ \\
\hline \multicolumn{7}{|l|}{ Demographic variable } \\
\hline Mean age, year (SD) & $65(12)$ & $64(12)$ & $68(11)$ & $66(12)$ & $68(12)$ & $68(10)$ \\
\hline Male (\%) & 45 & 52 & 38 & 44 & 39 & 48 \\
\hline \multicolumn{7}{|l|}{ Statin use ${ }^{*}$} \\
\hline Duration, month (range) & $24(6-42)$ & $15(4-28)$ & $22(6-41)$ & $16(5-32)$ & $23(7-41)$ & $16(6-32)$ \\
\hline $\begin{array}{l}\text { Daily prescribed dose, (mg, where } \\
\text { available) }\end{array}$ & 10 & 2.5 & 10 & $1.6(1-2)$ & 5 & $30(20-30)$ \\
\hline \multicolumn{7}{|l|}{ Comorbidity } \\
\hline Hepatic impairment (\%)† & 3 & 4 & 2 & 2 & 1 & 2 \\
\hline Renal impairment (\%)‡ & 25 & 31 & 22 & 25 & 15 & 28 \\
\hline $\mathrm{HbA} 1 \mathrm{c}>6.1 \%(\%)$ & 17 & 28 & 14 & 22 & 13 & 14 \\
\hline Hypertension (\%)§ & 25 & 53 & 55 & 28 & 60 & 57 \\
\hline Diabetes mellitus (\%)ף & 55 & 31 & 21 & 53 & 22 & 21 \\
\hline Cardiovascular disease $(\%)^{\star \star}$ & 32 & 30 & 27 & 25 & 28 & 40 \\
\hline Ischaemic heart disease (\%)†† & 18 & 17 & 16 & 14 & 14 & 20 \\
\hline 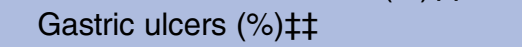 & 34 & 33 & 30 & 32 & 32 & 31 \\
\hline \multicolumn{7}{|l|}{ Lipid profile } \\
\hline TC $>1 \times$ ULN & $60 \%(2248 / 3773)$ & $68 \%(2547 / 3719)$ & $58 \%(1436 / 2467)$ & $60 \%(541 / 909)$ & $63 \%(261 / 419)$ & $40 \%(165 / 408)$ \\
\hline LDL-C >1× ULN & $71 \%(1639 / 2295)$ & $73 \%(3019 / 4118)$ & $64 \%(969 / 1524)$ & $69 \%(664 / 969)$ & $61 \%(147 / 243)$ & $51 \%(104 / 202)$ \\
\hline HDL-C <1×LLN & $16 \%(603 / 3375)$ & $18 \%(835 / 4638)$ & $13 \%(321 / 2288)$ & $15 \%(182 / 1235)$ & $13 \%(51 / 383)$ & $15 \%(76 / 497)$ \\
\hline TG >1×ULN & $54 \%(2320 / 4266)$ & $58 \%(2949 / 5056)$ & $45 \%(1274 / 2835)$ & $52 \%(665 / 1286)$ & $48 \%(223 / 463)$ & $46 \%(234 / 510)$ \\
\hline \multicolumn{7}{|c|}{ 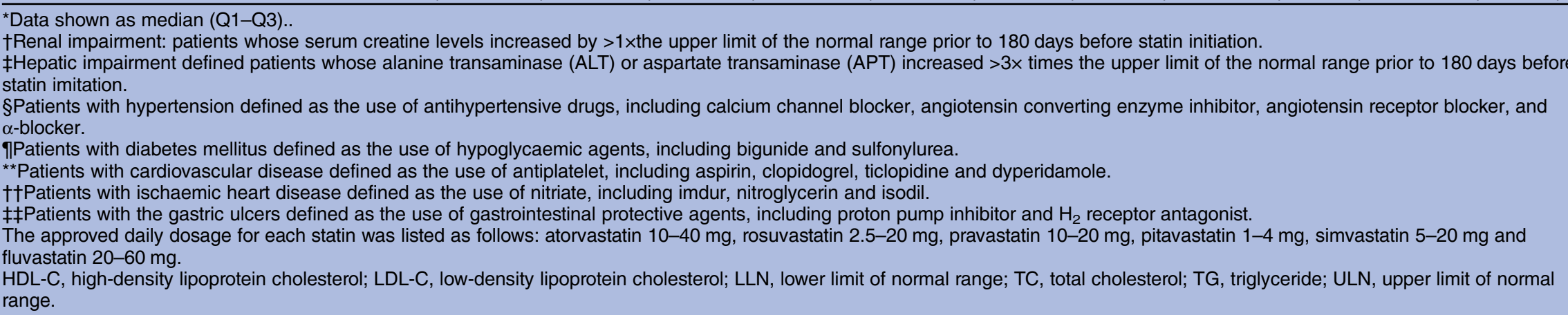 } \\
\hline
\end{tabular}


Table 2 Risks of muscle toxicity by various case definitions

\begin{tabular}{|c|c|c|c|c|}
\hline Outcome definitions & $\begin{array}{l}\text { Number of } \\
\text { cases with } \\
\text { events }\end{array}$ & $\begin{array}{l}\text { Number of } \\
\text { eligible } \\
\text { persons }\end{array}$ & Person-years & Incidence * $(95 \% \mathrm{Cl})$ \\
\hline $\begin{array}{l}\text { Base-case definition: criterion A (diagnosis of } \\
\text { 'myositis' or 'rhabdomyolysis') or criterion B } \\
\text { (CK >10xULN) }\end{array}$ & 43 & 18036 & 42193 & $1.02(0.76$ to 1.37$)$ \\
\hline With discontinuation & 10 & & & $0.24(0.13$ to 0.44$)$ \\
\hline With switching & 3 & & & 0.07 (0.02 to 0.22$)$ \\
\hline With renal dysfunction & 17 & & & $0.40(0.25$ to 0.65$)$ \\
\hline With hospitalisation & 17 & & & $0.40(0.25$ to 0.65$)$ \\
\hline Criterion $\mathrm{A}$ or $\mathrm{CK}>5 \times \mathrm{ULN}$ & 87 & 18036 & 42193 & 2.06 (1.67 to 2.54$)$ \\
\hline With discontinuation & 23 & & & 0.55 (0.36 to 0.82$)$ \\
\hline With switching & 7 & & & 0.17 (0.08 to 0.35$)$ \\
\hline With renal dysfunction & 39 & & & $0.92(0.68$ to 1.27$)$ \\
\hline With hospitalisation & 34 & & & 0.81 (0.58 to 1.13$)$ \\
\hline Criteria $A$ and $B$ & 4 & 18036 & 42193 & 0.09 (0.04 to 0.25$)$ \\
\hline With discontinuation & 3 & & & 0.07 (0.02 to 0.22$)$ \\
\hline With switching & 1 & & & $0.02(0.00$ to 0.17$)$ \\
\hline With renal dysfunction & 3 & & & 0.07 (0.02 to 0.22$)$ \\
\hline With hospitalisation & 4 & & & 0.09 (0.04 to 0.25$)$ \\
\hline Myositis (and criterion B) & 0 & & & - \\
\hline Rhabdomyolysis (and criterion B) & 4 & & & $0.09(0.04$ to 0.25$)$ \\
\hline Criterion $A$ and $C K>5 \times U L N$ & 8 & 18036 & 42193 & 0.19 (0.09 to 0.38$)$ \\
\hline With discontinuation & 3 & & & 0.07 (0.02 to 0.22$)$ \\
\hline With switching & 1 & & & $0.02(0.00$ to 0.17$)$ \\
\hline With renal dysfunction & 6 & & & $0.14(0.06$ to 0.32$)$ \\
\hline With hospitalisation & 7 & & & $0.17(0.08$ to 0.35$)$ \\
\hline Myositis (and CK > 5×ULN) & 0 & & & - \\
\hline Rhabdomyolysis (and CK $>5 \times$ ULN) & 8 & & & $0.19(0.09$ to 0.38$)$ \\
\hline Criterion A & 27 & 18036 & 42193 & $0.64(0.44$ to 0.93$)$ \\
\hline With discontinuation & 7 & & & $0.17(0.08$ to 0.35$)$ \\
\hline With switching & 3 & & & 0.07 (0.02 to 0.22$)$ \\
\hline With renal dysfunction & 9 & & & $0.21(0.11$ to 0.41$)$ \\
\hline With hospitalisation & 8 & & & 0.19 (0.09 to 0.38$)$ \\
\hline Myositis & 12 & & & $0.28(0.16$ to 0.50$)$ \\
\hline Rhabdomyolysis & 15 & & & $0.36(0.21$ to 0.59$)$ \\
\hline Criterion B & 20 & 18036 & 42193 & $0.47(0.31$ to 0.73$)$ \\
\hline With discontinuation & 6 & & & 0.14 (0.06 to 0.22$)$ \\
\hline With switching & 1 & & & $0.02(0.00$ to 0.17$)$ \\
\hline With renal dysfunction & 11 & & & $0.26(0.14$ to 0.47$)$ \\
\hline With hospitalisation & 13 & & & 0.31 (0.18 to 0.53$)$ \\
\hline CK >5xULN & 68 & & & 1.61 (1.27 to 2.04$)$ \\
\hline \multicolumn{5}{|l|}{ Baseline period } \\
\hline Base-case definition: 3 months & 43 & 18036 & 42193 & $1.02(0.76$ to 1.37$)$ \\
\hline 6 months & 40 & 16649 & 38645 & $1.04(0.76$ to 1.41$)$ \\
\hline 12 months & 31 & 13693 & 29387 & $1.05(0.74$ to 1.50$)$ \\
\hline \multicolumn{5}{|l|}{ Statin exposure status } \\
\hline Base-case definition: continuous exposure & 43 & 18036 & 42193 & $1.02(0.76$ to 1.37$)$ \\
\hline $\begin{array}{l}\text { Excluding stopping periods (days of statin } \\
\text { supply) }\end{array}$ & 39 & 18036 & 38027 & $1.03(0.75$ to 1.40$)$ \\
\hline
\end{tabular}

*Data shown as per 1000 person-years with $95 \% \mathrm{Cl}$.

Discontinuation was defined as no use of statin therapy in the 6 months following occurrence of muscle toxicity. Switching was defined as patients who initiated another statin in the following period. Renal dysfunction was defined as serum creatine increased above the upper limit of normal range within 3 days before or 7 days after occurrence of an event.

CK, creatine kinase; ULN, upper limit of the normal range.

of statins in clinical practice, because populations that participate in clinical trials are usually highly selected. ${ }^{26}$ Comparing our analysis to statistics reported in previous studies (mostly from the USA), the crude incidence of rhabdomyolysis ranged from 2.5 to $4.4 / 100000$ personyears among statin users, when identified by diagnosis 
Table 3 Risks of muscle toxicity among specific statin therapies

\begin{tabular}{llrr}
\hline Generic statin name & Number of cases with events & Person-years & Incidence $\dagger$ \\
\hline All statins & 43 & 42193 & $1.02(0.76$ to 1.37$)$ \\
Atorvastatin & 17 & 15776 & $1.08(0.67$ to 1.73$)$ \\
Rosuvastatin & 15 & 8655 & $1.73(1.04$ to 2.87$)$ \\
Pravastatin & 5 & 11121 & $0.45(0.19$ to 1.08$)$ \\
Pitavastatin & 3 & 2883 & $1.04(0.34$ to 3.23$)$ \\
Simvastatin & 2 & 2123 & $0.94(0.24$ to 3.77$)$ \\
Fluvastatin & 1 & 1635 & $0.61(0.09$ to 4.34$)$ \\
\hline
\end{tabular}

${ }^{*}$ Cases were defined by either criteria A (diagnosis of 'myositis' or 'rhabdomyolysis') or B (creatine kinase concentration $>10 \times$ the upper limit of the normal range).

†Data shown as per 1000 person-years with $95 \% \mathrm{Cl}$.

and laboratory results combined. ${ }^{22}{ }^{23}$ Since it is not applicable to compare the incidence of events defined by different parameters, we refined our event definition to consider statin discontinuation or switching and renal dysfunction or hospitalisation after the occurrence of an adverse event. Under this revised definition, we found the crude incidence of rhabdomyolysis to be 7.1 to $9.5 /$ 100000 person-years (3-4 cases per 42193 personyears). The incidence of rhabdomyolysis might differ among studies because the proportion of patients with risk factors is apparently different between the populations. Some case reports indicated that factors related to statin-associated muscle toxicity included old age, female gender, low body mass index and diabetes mellitus, ${ }^{27}$ characteristics that were common among patients in our study. However, evidence showed no increase in the rate of adverse events in Asian patients taking either lower or higher doses of statin, ${ }^{28}$ despite racial differences in the pharmacokinetics of rosuvastatin between Asians and Caucasians. $^{29}$ After all the controversy over racial differences in pharmacokinetics and the clinical outcomes of statin (particularly rosuvastatin) use, the majority of prescribed doses were at the lower limits of approved dosage levels, implying comparable potency among statins at the lowest effective dose. Taking dosage levels into account, statins were well tolerated in the Japanese population, with a similar incidence of rhabdomyolysis as that reported in Caucasian populations.

Using the claims database to evaluate potential drug interactions with statins is a useful complement to the limited information available from voluntary reports and clinical trials. A recent study found that the use of against label statin-fibrate combination therapy is decreasing annually, but that use of this therapy persists in the USA. ${ }^{30}$ In the present study, only $2.8 \%$ of patients had been prescribed concomitant statin-fibrate therapy (data not shown). Although combination therapy may be attractive for patients with lipid disorders and without muscle complaints, the low prevalence of concomitant fibrate use implies that it is 'generally contraindicated',

Table 4 Characteristics of patients exhibiting criteria A versus $B$

\begin{tabular}{|c|c|c|c|}
\hline & Criterion $A^{*}(\mathrm{~N}=27)$ & Criterion $\mathrm{B}^{*}(\mathrm{~N}=20)$ & p Value \\
\hline \multicolumn{4}{|l|}{ Demographic variable } \\
\hline Male (\%) & 52 & 55 & 0.83 \\
\hline Mean age, years (SD) & $60(15)$ & $69(11)$ & 0.07 \\
\hline Statin use at the event (\%) & & & 1.00 \\
\hline Atorvastatin & 37 & 35 & \\
\hline Fluvastatin & 4 & 0 & \\
\hline Pitavastatin & 4 & 10 & \\
\hline Pravastatin & 11 & 15 & \\
\hline Rosuvastatin & 37 & 35 & \\
\hline Simvastatin & 7 & 5 & \\
\hline Mean interval after initiating Statin, month (SD) & $18(13)$ & $19(20)$ & 0.98 \\
\hline \multicolumn{4}{|l|}{ Laboratory information } \\
\hline SCr $>1 \times$ ULN & $56 \%(9 / 16)$ & $58 \%(11 / 19)$ & 0.92 \\
\hline BUN >1×ULN & $44 \%(7 / 16)$ & $50 \%(10 / 20)$ & 0.71 \\
\hline APT $>1 \times$ ULN & $56 \%(9 / 16)$ & $80 \%(16 / 20)$ & 0.12 \\
\hline ALT >1×ULN & $38 \%(6 / 16)$ & $65 \%(13 / 20)$ & 0.10 \\
\hline
\end{tabular}

${ }^{*}$ Criterion $\mathrm{A}$ is defined by diagnosis of 'myositis' or 'rhabdomyolysis' and criterion $\mathrm{B}$ is defined by creatine kinase (CK) concentration $>10 \times$ times the upper limit of the normal range. Four cases met both criteria $A$ and $B$.

ALT, alanine transaminase; APT, aspartate transaminase; BUN, blood urea nitrogen; SCr, serum creatinine; ULN, upper limit of the normal range. 
Table 5 Risk of muscle toxicity from concomitant use of interacting drugs

\begin{tabular}{lll} 
& $\begin{array}{l}\text { Concomitant use } \\
\text { of interacting drugs }\end{array}$ & $\begin{array}{l}\text { No concomitant use } \\
\text { of interacting drugs }\end{array}$ \\
\hline $\mathrm{N}$ & 2430 & 15606 \\
Person-year & 1776 & 40418 \\
Number of events & 3 & 40 \\
Proportion of total events (\%) & 0.12 & 0.26 \\
Incidence per 1000 person-years (95\% CI) & 1.69 (0.54 to 5.24) & $0.99(0.73$ to 1.35) \\
\hline${ }^{*}$ The number of patients who were exposed to specific interacting drugs in this study were as follows: benzafibrate (256), fenofibrate (262), \\
clinofibrate (1), clarithromycin (1,688), erythromycin (77), telithromycin (2), fluconazole (22), itraconazole (125), fosfluconazole (31), \\
voriconazole (11), cyclosporine (66), amiodarone (93), saquinavir/ritonavir (0), atazanavir (0), etraririne (0) and efavirenz (0).
\end{tabular}

and that clinical practitioners are aware of the risks. Whether the risk is due to drug interactions remains controversial, $^{31} 32$ but our study points to a higher incidence of muscle toxicity among patients taking interacting drugs. However, a low number of person-years of interacting drug use observations corresponded to a lack of statistical significance; this low prevalence of concomitant drug use might reduce the detection of muscle toxicity in current practice.

Our findings showed that the incidence of muscle toxicity varied according to the definition of an adverse event, suggesting that the infrastructure in which information is stored might greatly affect assessments of safe medical practice. The majority of cases of discontinuation or switching of statins resulted in hospitalisation accompanied by acute changes in renal function. However, the outcome from severe muscle toxicity could not be ascertained since there is no information of death record in the database. Although diagnosis-based measures are inherently limited by weaknesses of administrative claims diagnostic data, including inaccuracy and incompleteness of discharge diagnoses, ${ }^{33}$ the prescription claims have been shown to be fairly accurate and complete. ${ }^{34}$ Thus, laboratory information was used as a surrogate indicator in this study when there was no corresponding treatment. The comparative characteristics between our two criteria imply that safety measures should be incorporated for a conservative interpretation of laboratory information. In order to avoid overestimating the risk of muscle toxicity, we identified the accompanying condition by both diagnosis and drugs when an event occurred, and excluded cases that were less likely to be related to statin use. Furthermore, the MDV database provided objective information that would not only complement the limitations of the claims database, but that would also provide the potential to monitor pharmacological responses to therapeutic interventions using biomarkers. In addition, the MDV plans to enrol up to 100 medical facilities to improve the applicability of using electronic health information in the near future. Limits to investigations imposed by small sample sizes could be overcome, and associations between the risk of adverse events and the use of statins with and without interacting drugs could be explored through this strategy. However, hospital-based data collection would be threatened by lost follow-up and lack of enrolment information such as patients' date of birth and geographic region. Since the database used in this study did not have enough power to evaluate rare events in Japanese clinical practice, the accessibility of a national claims database is expected to provide a complement to this limitation. In addition, the MIHARI project plans to aggregate information from 10 university hospitals (the Sentinel database) to confirm the signal with objective information based on appropriate methodology. Since the validity of claims-based information is often questioned, studies should be conducted to verify the validity ${ }^{35}$ and explore the applicability of this information. From a regulatory perspective, great potential exists for evaluating risks for a population using validated information, with the goal of making efficient use of the resource. By using unique identification or indirect identifiers such as patient's date of birth, sex, hospital identification number, admission date and discharge date, linkage between different databases is commonly used when clinical trial designs are not applicable. ${ }^{36-38}$ As the accessibility of healthcare information in Japan is strictly regulated by privacy protection, further discussion would be necessary to balance privacy protection with an active drug safety monitoring system.

\section{CONCLUSIONS}

The present study provides evidence of incidence of muscle toxicity with statin use, identified by diagnosis and objective laboratory information obtained from the claims database in Japan. Since the definition of safety measures varies according to the infrastructure of data resources, researchers should use caution when interpreting risk information that provides answers to uncertainties addressed before drug approval. While the use of combination therapy is relatively low in patients with lipid disorders and without muscle complaints, the risks attributed to drug interactions in statin users require further exploration.

\section{Author affiliations}

${ }^{1}$ Institute of Clinical Pharmacy and Pharmaceutical Sciences, National Cheng Kung University, Tainan, Taiwan

${ }^{2}$ Laboratory of Pharmaceutical Regulatory Science, Graduate School of Pharmaceutical Sciences, The University of Tokyo, Tokyo, Japan ${ }^{3}$ Sugiyama Laboratory, RIKEN Innovation Center, Research Cluster for Innovation, RIKEN, Kanagawa, Japan 
${ }^{4}$ Pharmacy Department, NTT Medical Center Tokyo, Tokyo, Japan

${ }^{5}$ Department of Public Health and Epidemiology, Meiji Pharmaceutical University, Tokyo, Japan

Acknowledgements We would like to thank Ms Kaori Ota and Mr Masaki Nakamura, staff of Medical Data Vision Co. Ltd (Tokyo Japan), for their technical support in using the commercial electronic healthcare database.

Contributors MA was the principal investigator for the grant. MK, TO and MA conceived the study. All authors contributed to the study design. TO and MA were responsible for obtaining the data. $\mathrm{C}-\mathrm{HC}$ and $\mathrm{MK}$ conducted the initial data analysis. All authors contributed to decisions on the interpretation of results. $\mathrm{C}-\mathrm{HC}, \mathrm{KM}$ and MA contributed to the drafting of the manuscript. All authors approved the final version of the manuscript prior to submission.

Funding This study was supported by a research grant (grant number 11-8003) from the Pfizer Health Research Foundation (Tokyo Japan). The sponsor had no role in the study design, data collection, data analysis, data interpretation or approval of the manuscript.

Competing interests None.

Ethics approval Meiji Pharmaceutical University.

Provenance and peer review Not commissioned; externally peer reviewed.

Data sharing statement Technical appendix, statistical code and dataset available from the corresponding author (makazawa@my-pharm.ac.jp). Informed consent for data sharing was not obtained but the presented data are anonymised and risk of identification is low.

\section{REFERENCES}

1. Grundy SM, Cleeman JI, Merz CN, et al. Implications of recent clinical trials for the National Cholesterol Education Program Adult Treatment Panel III guidelines. Circulation 2004;110:227-39.

2. Davidson $M H$. Safety profiles for the HMG-CoA reductase inhibitors: treatment and trust. Drugs 2001;61:197-206.

3. Shepherd J, Vidt DG, Miller E, et al. Safety of rosuvastatin: update on 16,876 rosuvastatin-treated patients in a multinational clinical tria program. Cardiology 2007;107:433-43.

4. Classen DC, Pestotnik SL, Evans RS, et al. Adverse drug events in hospitalized patients. Excess length of stay, extra costs, and attributable mortality. JAMA 1997;277:301-6.

5. Rowan C, Brinker AD, Nourjah P, et al. Rhabdomyolysis reports show interaction between simvastatin and CYP3A4 inhibitors. Pharmacoepidemiol Drug Saf 2009;18:301-9.

6. Matsuzawa $\mathrm{Y}, \mathrm{Kita} \mathrm{T}$, Mabuchi $\mathrm{H}$, et al. Sustained reduction of serum cholesterol in low-dose 6-year simvastatin treatment with minimum side effects in 51,321 Japanese hypercholesterolemic patients. Circ J 2003;67:287-94

7. Saito M, Hirata-Koizumi M, Urano $\mathrm{T}$, et al. A literature search on pharmacokinetic drug interactions of statins and analysis of how such interactions are reflected in package inserts in Japan. $J$ Clin Pharm Ther 2005;30:21-37.

8. Arnold FL, Kusama M, Ono S. Exploring differences in drug doses between Japan and Western countries. Clin Pharmacol Ther 2010;87:714-20.

9. Wang P. Statin dose in Asians: is pharmacogenetics relevant? Pharmacogenomics 2011;12:1605-15.

10. Cziraky MJ, Willey VJ, McKenney JM, et al. Statin safety: an assessment using an administrative claims database. Am J Cardiol 2006;97:61C-8C

11. Molokhia M, McKeigue $P$, Curcin V, et al. Statin induced myopathy and myalgia: time trend analysis and comparison of risk associated with statin class from 1991-2006. PLOS ONE 2008;3:e2522.

12. Robb MA, Racoosin JA, Sherman RE, et al. The US Food and Drug Administration's Sentinel Initiative: expanding the horizons of medical product safety. Pharmacoepidemiol Drug Saf 2012;(Suppl 1):9-11.

13. Pharmaceuticals and Medical Devices Agency. MIHARI project. Tokyo, Japan, 2011. http://www.pmda.go.jp/english/service/ mihari_project.html (accessed 31 Aug 2012).
14. Kimura T, Matsushita $\mathrm{Y}$, Yang $\mathrm{YH}$, et al. Pharmacovigilance systems and databases in Korea, Japan, and Taiwan. Pharmacoepidemiol Drug Saf 2011;20:1237-45.

15. Food and Drug Administration. FDA drug safety communication: important safety label changes to cholesterol-lowering statin drugs. 2012. http://www.fda.gov/Drugs/DrugSafety/ucm293101.htm (accessed 31 Aug 2012)

16. Hashikata $\mathrm{H}$, Harada $\mathrm{KH}$, Kagimura $\mathrm{T}$, et al. Usefulness of a large automated health records database in pharmacoepidemiology. Environ Health Prev Med 2011;16:313-19.

17. Urushihara $\mathrm{H}$, Taketsuna $\mathrm{M}$, Liu $\mathrm{Y}$, et al. Increased risk of acute pancreatitis in patients with type 2 diabetes: an observational study using a Japanese hospital database. PLoS One 2012; 7:e53224.

18. Japanese Ethical Guidelines for Epidemiological Research. (In Japanese). 2008. http://www.mhlw.go.jp/general/seido/kousei/ i-kenkyu/ekigaku/0504sisin.html (accessed 31 Aug 2012).

19. Okuno J, Yanagi $\mathrm{H}$, Tomura $\mathrm{S}$, et al. Compliance and medication knowledge among elderly Japanese home-care recipients. Eur $J$ Clin Pharmacol 1999:55:145-9.

20. Ohta Y, Tsuchihashi T, Onaka U, et al. Long-term compliance of salt restriction and blood pressure control status in hypertensive outpatients. Clin Exp Hypertens 2010;32:234-8.

21. Pharmaceutical and Medical Devices Agency. PMDA package insert database for medicine product approved in Japan. 2012 (in Japanese). http://www.info.pmda.go.jp/info/iryo_index.html (accessed 31 Aug 2012).

22. Graham DJ, Staffa JA, Shatin D, et al. Incidence of hospitalized rhabdomyolysis in patients treated with lipid-lowering drugs. JAMA 2004;292:2585-90.

23. Amend $\mathrm{KL}$, Landon J, Thyagarajan V, et al. Incidence of hospitalized rhabdomyolysis with statin and fibrate use in an insured US population. Ann Pharmacother 2011;45:1230-9.

24. McKenney JM, Davidson MH, Jacobson TA, et al. Final conclusions and recommendations of the National Lipid Association Statin Safety Assessment Task Force. Am J Cardiol 2006;97:89C-94C.

25. Seino $\mathrm{Y}$, Nanjo K, Tajima N, et al. Report of the committee on the classification and diagnostic criteria of diabetes mellitus (in Japanese). J Jpn Diabetes Soc 2010;53:450-7.

26. Armitage J. The safety of statins in clinical practice. Lancet 2007;370:1781-90.

27. Shek A, Ferrill MJ. Statin-fibrate combination therapy. Ann Pharmacother 2001;35:908-17.

28. Liao JK. Safety and efficacy of statins in Asians. Am J Cardiol 2007;99:410-14.

29. Lee E, Ryan S, Birmingham B, et al. Rosuvastatin pharmacokinetics and pharmacogenetics in white and Asian subjects residing in the same environment. Clin Pharmacol Ther 2005;78:330-41.

30. Alford JC, Saseen JJ, Allen RR, et al. Persistent use of against-labe statin-fibrate combinations from 2003-2009 despite United States Food and Drug Administration dose restrictions. Pharmacotherapy 2012;32:623-30.

31. Bottorff MB. Statin safety and drug interactions: clinical implications. Am J Cardiol 2006;97:27C-31C

32. Rowan CG, Brunelli SM, Munson J, et al. Clinical importance of the drug interaction between statins and CYP3A4 inhibitors: a retrospective cohort study in The Health Improvement Network. Pharmacoepidemiol Drug Saf 2012;21:494-506.

33. Iezzoni LI. Assessing quality using administrative data. Ann Intern Med 1997;127:666-74.

34. Sloan KL, Sales AE, Liu CF, et al. Construction and characteristics of the RxRisk-V: a VA-adapted pharmacy-based case-mix instrument. Med Care 2003;41:761-74.

35. Cheng CL, Kao YH, Lin SJ, et al. Validation of the National Health Insurance Research Database with ischemic stroke cases in Taiwan. Pharmacoepidemiol Drug Saf 2011;20:236-42.

36. Tuckuviene R, Christensen AL, Helgestad J, et al. Paediatric arteria ischaemic stroke and cerebral sinovenous thrombosis in Denmark 1994-2006: a nationwide population-based study. Acta Paediatr 2011;100:543-9.

37. Chang $\mathrm{CH}$, Toh S, Lin JW, et al. Cancer risk associated with insulin glargine among adult type 2 diabetes patients-a nationwide cohort study. PLOS ONE 2011;6:e21368.

38. Weintraub WS, Grau-Sepulveda MV, Weiss JM, et al. Comparative effectiveness of revascularization strategies. $N$ Engl J Med 2012;366:1467-76. 